\title{
Facing $\mathrm{SpO}_{2}$ and $\mathrm{SaO}_{2}$ discrepancies in ICU patients: is the perfusion index helpful?
}

\author{
Mark Thijssen $^{1}\left[\right.$ [ Loes Janssen ${ }^{2} \cdot$ Jos le Noble $^{1} \cdot$ Norbert Foudraine $^{1}$
}

Received: 4 April 2019 / Accepted: 3 August 2019 / Published online: 7 August 2019

(c) The Author(s) 2019

\begin{abstract}
Peripheral oxygen saturation $\left(\mathrm{SpO}_{2}\right)$ measured by pulse oximetry is an unreliable surrogate marker for arterial oxygenation $\left(\mathrm{SaO}_{2}\right)$ in critically ill patients. We hypothesized that a higher perfusion index (PFI) would be associated with better accuracy of $\mathrm{SpO}_{2}$ measurement. We retrospectively collected $\mathrm{SaO}_{2}, \mathrm{SpO}_{2}$, and PFI data for each arterial blood gas (ABG) analysis in a cohort of intensive care unit patients. PFI was categorised as low $(\mathrm{PFI}<1.0)$, intermediate $(1.0 \leq \mathrm{PFI} \leq 2.5)$, or high (PFI > 2.5). The correlation between $\mathrm{SpO}_{2}$ and $\mathrm{SaO}_{2}$ was studied using Pearson's correlation. The Bland-Altman plot was used to analyse the agreement between $\mathrm{SpO}_{2}$ and $\mathrm{SaO}_{2}$. Furthermore, the correlation between the $\left(\mathrm{SpO}_{2}-\mathrm{SaO}_{2}\right)$ difference and PFI was assessed. The level of (dis)agreement was calculated for the three PFI categories separately. Overall, 281 patients and 1281 data points were analysed. There was a significant correlation between $\mathrm{SaO}_{2}$ and $\mathrm{SpO}_{2}(\mathrm{r}=0.69, \mathrm{p}<0.01)$. The Bland-Altman analysis revealed a mean difference between $\mathrm{SaO}_{2}$ and $\mathrm{SpO}_{2}$ of $0.2 \%$ with limits of agreement of $\pm 6 \%$ $(\mathrm{SD} \pm 2 \%)$. The correlation between the PFI and the $\left(\mathrm{SpO}_{2}-\mathrm{SaO}_{2}\right)$ difference was low; the $\left(\mathrm{SpO}_{2}-\mathrm{SaO}_{2}\right)$ difference improved only marginally with higher PFI values. The accuracy of pulse oximetry for estimating arterial oxygenation was moderate and improved little with increasing PFI values. Thus, the additive value of PFI in clinical decision making is limited. Therefore, we advise performing an $\mathrm{ABG}$ before adjusting fraction of inspired oxygen $\left(\mathrm{FiO}_{2}\right)$ settings.
\end{abstract}

Keywords Critical illness · Intensive care unit · Oxygen saturation · Perfusion index · Pulse oximetry

\section{Introduction}

Pulse oximetry is routinely used to monitor peripheral oxygen saturation $\left(\mathrm{SpO}_{2}\right)$ as a surrogate marker for arterial oxygen saturation $\left(\mathrm{SaO}_{2}\right)$. Strict guidelines regarding an optimum target $\mathrm{SpO}_{2}$ are lacking. However, most clinicians will aim for $\mathrm{SpO}_{2}$ values between 88 and $96 \%$, depending on the clinical situation, and might surmise that the real arterial

Mark Thijssen

m.thijssen@zuyderland.nl

Loes Janssen

loes.janssen@mmc.nl

Jos le Noble

jlenoble@maastrichtuniversity.nl

Norbert Foudraine

nfoudraine@ viecuri.nl

1 Department of Intensive Care, VieCuri Medical Center, Tegelseweg 210, 5912BL Venlo, The Netherlands

2 Department of Statistics and Epidemiology, VieCuri Medical Center, Tegelseweg 210, 5912BL Venlo, The Netherlands oxygenation is above the measured values of the pulse oximeter [1]. However, one can erroneously accept lower arterial oxygenations than surmised. Although it is a useful method for monitoring intensive care unit (ICU) patients, it has limitations. In the critically ill, complex physiological disturbances such as altered blood flow, acid-base disturbances, and abnormalities in temperature regulation may occur; these affect the oxyhaemoglobin dissociation curve and might further complicate the interpretation of the $\mathrm{SpO}_{2}$ [2]. Moreover, the accuracy of a pulse oximeter is reduced in patients with low perfusion status, sepsis, and vasopressor use [3,4]. Consequently, the new oximeters can calculate the perfusion index (PFI) from a pulsatile photo plethysmography signal and indirectly measure the perfusion variations. The PFI may function as a marker for peripheral perfusion and resembles vasomotor tone, with low and high PFI values indicating perfusion below and above average, respectively [5-9]. In routine ICU practice, the PFI could possibly be used as a surrogate marker for the accuracy of measured $\mathrm{SpO}_{2}[10]$. 
The primary objective of our study was to assess whether, in a cohort of ICU patients, the use of the PFI in daily practice contributes to the accuracy of pulse oximetry. We hypothesized that higher PFI values would be associated with more accurate $\mathrm{SpO}_{2}$ measurement. Therefore, we studied the relationship between $\mathrm{SaO}_{2}$ and $\mathrm{SpO}_{2}$, and how this depended on the PFI. Furthermore, as a secondary objective, we tested whether multiple variables (temperature, $\mathrm{MAP}, \mathrm{pH}$, lactate, and inotropic drug use) influenced the $\left(\mathrm{SpO}_{2}-\mathrm{SaO}_{2}\right)$ difference and PFI.

\section{Materials and methods}

We performed a retrospective study in a level 2 mixed ICU of a single-site teaching hospital. Patients were included in the study between May 2015 and September 2015. All admitted ICU patients on supplemental oxygen therapy, with at least one arterial blood gas (ABG) analysis and concomitantly measured $\mathrm{SpO}_{2}$, were eligible for inclusion.

\subsection{Data collection}

Data regarding patient characteristics, admission diagnosis, length of stay (LOS), days on non-invasive/invasive mechanical ventilation, $\mathrm{SpO}_{2}$, PFI, and variables that could potentially influence pulse oximetry measurements such as central body temperature, mean arterial pressure (MAP), $\mathrm{pH}$, lactate, and inotropic drug use were retrieved from the electronic patient data management system (EPD). EPD data verification was performed by the research nurse. In patients treated with invasive ventilation, all ABG samples were included until extubation. In all other cases, one daily ABG was included until a maximum of 3 days after ICU admittance in order to limit the total amount of data, under the assumption that any further data would not strongly influence outcomes.

$\mathrm{SaO}_{2}, \mathrm{PaO}_{2}, \mathrm{pH}$, and lactate were measured with a blood gas analyser (ABL 800 Flex, Radiometer, Copenhagen, Denmark) in the local clinical chemistry department; this method is considered the gold standard.

\subsection{Pulse oximeters}

$\mathrm{SpO}_{2}$ was measured continuously; however, for this study, $\mathrm{SpO}_{2}$ registered only at the time of blood withdrawal using the Philips M1191BL finger probe (Philips Healthcare, Eindhoven, The Netherlands) and the Philips M1194A ear probe (Philips Healthcare, Eindhoven, The Netherlands). According to the manufacturer, these pulse oximeters have an accuracy of $\pm 2.5 \%$ and $\pm 4 \%$ root mean square (RMS), respectively [11]. Both pulse oximeters were connected to the Philips MP70 monitor (Philips Healthcare, Eindhoven, The Netherlands) [10].

\subsection{Statistical analysis}

All statistical analyses were performed with IBM SPSS Statistics version 23 (IBM Corp. Released 2015. IBM SPSS Statistics for Windows, Version 23.0. Armonk, NY: IBM Corp.) Continuous data are presented as a mean if normally distributed and as a median otherwise. Categorical data are presented as percentages.

\subsubsection{Accuracy of pulse oximetry}

We used the Pearson correlation coefficient (r) to express the relationship between $\mathrm{SaO}_{2}$ and $\mathrm{SpO}_{2}$. The Bland-Altman plot was used to graphically represent this relationship [12]. With this method, the difference between $\mathrm{SpO}_{2}$ and $\mathrm{SaO}_{2}$ ( $\Delta$ Sat) was plotted against the average of $\mathrm{SpO}_{2}$ and $\mathrm{SaO}_{2}$. A positive value indicated that the measured $\mathrm{SaO}_{2}$ was lower than $\mathrm{SpO}_{2}$. Furthermore, regression analysis was performed to determine whether there was positive, negative, or no correlation between the bias and the average [13]. The Pearson correlation coefficient was used to express the possible association of temperature, $\mathrm{pH}$, lactate, and MAP with $\Delta$ Sat. Furthermore, a Student's $t$ test was performed to test the association of sex with $\Delta$ Sat. One-way ANOVA was used to test the association of inotropes with $\Delta$ Sat.

\subsubsection{The perfusion index}

To evaluate the relationship between the (skewed) PFI and $\Delta$ Sat, the Spearman correlation coefficient was calculated. Subsequently, we determined whether the PFI is a reliable surrogate for the accuracy of the pulse oximeter. We categorised PFI values as low $(\mathrm{PFI}<1.0)$, intermediate $(1.0 \leq \mathrm{PFI} \leq 2.5)$, or high $(\mathrm{PFI}>2.5)[5,7,10]$. Accuracy was visualised by plotting $\Delta$ Sat against the PFI. In addition, we calculated the percentages of measurements exceeding various limits of agreement for the three PFI categories.

The study protocol was approved by the local Ethics Review Board and only the primary investigator was able to link the study data with the patient data [14].

\section{Results}

A total of 320 patients were eligible for the study. The data from 39 patients were missing and the remaining 281 were included in the analysis, resulting in 1281 data points. Baseline patient characteristics are shown in Table 1.

On average, $\mathrm{SaO}_{2}$ and $\mathrm{SpO}_{2}$ correlated moderately $(\mathrm{r}=0.69, \mathrm{p}<0.01, \mathrm{n}=1281)$. The Bland-Altman plot 
Table 1 Standard patient characteristics

\begin{tabular}{|c|c|}
\hline $\begin{array}{l}\text { Patient characteristics } \\
(\mathrm{n}=281)\end{array}$ & $\mathrm{n}(\%)$ \\
\hline \multicolumn{2}{|l|}{ Sex } \\
\hline Male & $177(63)$ \\
\hline Female & $104(37)$ \\
\hline Age in years (SD) & $65(15)$ \\
\hline \multicolumn{2}{|l|}{ Diagnosis at admittance* } \\
\hline Pneumonia & $31(11.0)$ \\
\hline Cardiac arrest & $28(10.0)$ \\
\hline Trauma & $23(8.2)$ \\
\hline GI bleeding & $18(6.4)$ \\
\hline Sepsis & $16(5.7)$ \\
\hline COPD exacerbation & $15(5.3)$ \\
\hline Length of stay in days (range) & $2(0-67)$ \\
\hline Days intubated (range) & $3(0-58)$ \\
\hline \multicolumn{2}{|l|}{ Inotropic use } \\
\hline Norepinephrine & $102(36)$ \\
\hline Dobutamine & $10(4)$ \\
\hline Combination & $19(7)$ \\
\hline APACHE IV* & $87(35)$ \\
\hline $\mathrm{SaO}_{2}$ in $\%(\mathrm{SD})^{*}$ & $96.1(3.8)$ \\
\hline $\mathrm{SpO}_{2}$ in $\%(\mathrm{SD})^{*}$ & $96.3(3.9)$ \\
\hline PFI in \% (range)* & $1.4(0.1-19.2)$ \\
\hline
\end{tabular}

Continuous data are presented as means (SD) or medians (interquartile range). Categorical data are presented as percentages

*GI Bleeding gastrointestinal bleeding, COPD chronic obstructive pulmonary disease, APACHE IV acute health and chronic health evaluation IV, $\mathrm{SaO}_{2}$ arterial oxygen saturation, $\mathrm{SpO}_{2}$ peripheral oxygen saturation, $P F I$ perfusion index

revealed a mean $\Delta$ Sat of $0.21 \%$, with a standard deviation (SD) of $3.04 \%$ (Fig. 1). Limits of agreement within 2 SDs of the mean showed a $\Delta$ Sat of $-5.75 \%$ and $+6.17 \%$. Furthermore, a non-significant linear regression between the bias $(\Delta$ Sat $)$ and the magnitude of the measurements (mean of $\mathrm{SaO}_{2}$ and $\left.\mathrm{SpO}_{2}\right)$ was found $(\mathrm{F}(1,1279)=1.44$, $\mathrm{p}<0.23$ ), with a $\mathrm{R}^{2}$ of 0.001 , indicating no correlation between the bias and the error and verifying reliable use of the Bland-Altman analysis.

The mean $\Delta$ Sat was not different between males and females $(0.27$ versus $0.13, p=0.42)$ and was not associated with inotropic drugs use $(F(3,1277)=0.94, p=0.42)$. In addition, other independent variables showed no or poor association with $\Delta$ Sat $(\mathrm{pH}: \mathrm{r}=-0.18, \mathrm{p}<0.01$; lactate: $r=0.02, p=0.60 ;$ MAP: $r=-0.006, p=0.82$ ).

In the case of the largest measured $\Delta$ Sat, we measured both methaemoglobin (MetHb) and carboxyhaemoglobin $(\mathrm{COHb})$. All values of combined MetHb plus $\mathrm{COHb}$ were under $2 \%$ (data not shown).

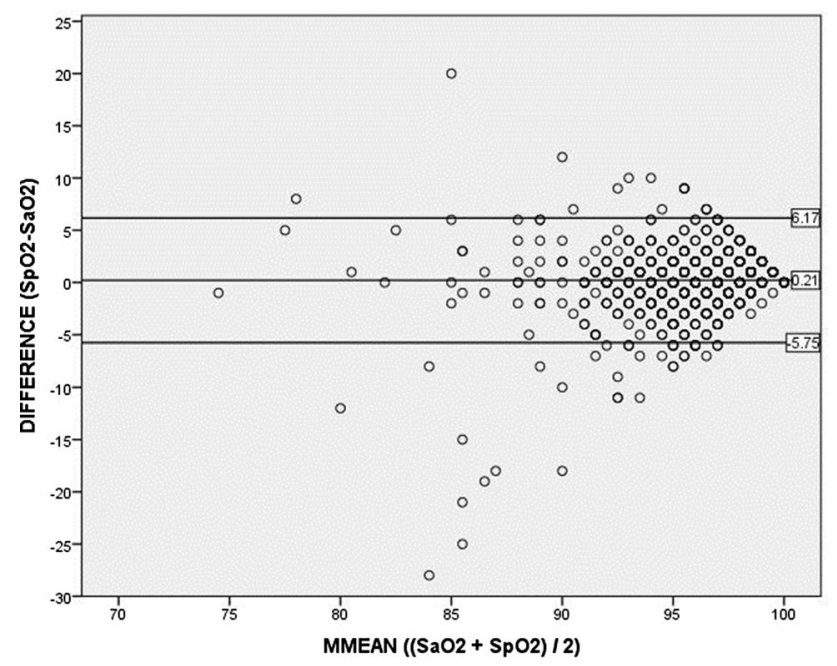

Fig. 1 Bland-Altman plot, in which the difference between $\mathrm{SpO}_{2}$ and $\mathrm{SaO}_{2}$ is plotted against their average. The mean difference is $0.21 \%$ (middle line), with a SD (precision) of 3.04\%. Limits of agreement were calculated using mean $\pm(1.96 * \mathrm{SD})$, resulting in an upper limit of $6.17 \%$ and a lower limit of $-5.75 \%$ (outer lines)

\subsection{The perfusion index}

PFI and $\Delta$ Sat correlated weakly $(\mathrm{r}=-0.17, \mathrm{p}<0.01)$, indicating a slightly better agreement between $\mathrm{SaO}_{2}$ and $\mathrm{SpO}_{2}$ with increasing PFI (Fig. 2, Table 2). A large proportion of values exceeded the limits of agreement, which did not decrease drastically with increasing PFI. For example, even with a PFI $>2.5$, which is generally considered adequate, $15.9 \%$ of all measurements still showed a difference between $\mathrm{SpO}_{2}$ and $\mathrm{SaO}_{2}$ of more than $2 \%$ (Table 2). Thus, the effect of PFI on $\Delta$ Sat is limited.

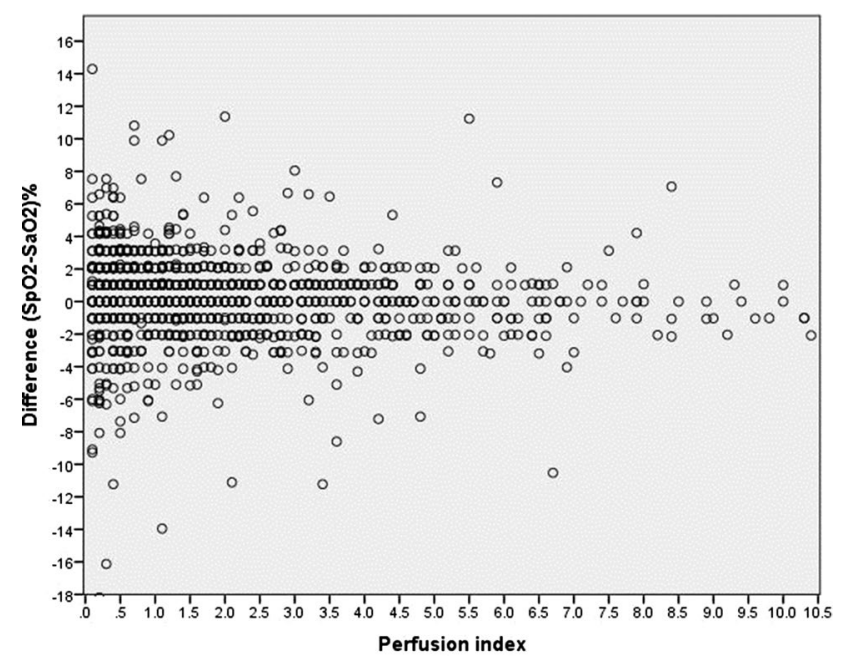

Fig. 2 The $\Delta$ Sat as percentages $\left(\left(\mathrm{SpO}_{2}-\mathrm{SaO}_{2} / \mathrm{SaO}_{2}\right) \times 100 \%\right)$ plotted against the PFI 
Table 2 Percentages and datapoints (n) of measurements exceeding varying limits of agreements (columns) for the various PFI categories (rows)

\begin{tabular}{lllll}
\hline$\Delta \mathrm{Sat}_{\left(\mathrm{SpO}_{2-}-\right.}$ & $>1 \%$ & $>2 \%$ & $>3 \%$ & $>4 \%$ \\
$\left.\mathrm{SaO}_{2}\right)$ & & & & \\
$\mathrm{PFI}$ & & & & \\
\hline$<1.0$ & $52.8 \%(246)$ & $30.7 \%(143)$ & $18.7 \%(87)$ & $10.5 \%(49)$ \\
$1.0-2.5$ & $38.3 \%(153)$ & $18.3 \%(73)$ & $8.5 \%(34)$ & $4.8 \%(19)$ \\
$>2.5$ & $36.2 \%(130)$ & $15.9 \%(57)$ & $6.9 \%(25)$ & $4.1 \%(15)$ \\
\hline
\end{tabular}

\section{Discussion}

We performed a retrospective, single-centre study to demonstrate the contribution of the PFI to the accuracy of pulse oximetry in critically ill adult patients. The main finding of our study is that even with high PFI values, the accuracy of pulse oximetry in estimating arterial oxygenation remains moderate; therefore, the additive value of PFI is limited. In our study, pulse oximetry and $\mathrm{SaO}_{2}$ measurements correlated moderately $(r=0.69)$. Even when the outliers were removed, results remained identical. As the correlation coefficient found between $\mathrm{SaO}_{2}$ and $\mathrm{SpO}_{2}$ was 0.69 , only $50 \%$ of the variability of $\mathrm{SpO}_{2}$ could be explained by the difference in $\mathrm{SaO}_{2}$. This is partly explained by a $3 \%$ variability of the pulse oximeter, compared to a variability of $0.2 \%$ of the blood gas analyser. This indicates that various other factors, such as acid-base disorders or factors contributing to macro- or microcirculation, might play a role in determining the absolute values of both $\mathrm{SpO}_{2}$ and $\mathrm{SaO}_{2}$. This comparison between $\mathrm{SpO}_{2}$ and $\mathrm{SaO}_{2}$ was also substantiated in the Bland-Altman plot, showing only moderate accuracy with varying clinical conditions.

Our findings regarding a discrepancy between $\mathrm{SaO}_{2}$ and $\mathrm{SpO}_{2}$ measurements are in line with the results of previous studies conducted with similar cohorts of critically ill patients. Perkins et al. demonstrated that changes in $\mathrm{SpO}_{2}$ do not reliably predict changes in $\mathrm{SaO}_{2}$, and neither anaemia nor acidosis altered the relationship between $\mathrm{SpO}_{2}$ and $\mathrm{SaO}_{2}$ [15]. We only found a small and weak negative correlation between the $\mathrm{pH}$ and $\Delta$ Sat. In a cohort of ventilator-dependent patients, Seguin et al. showed that $\mathrm{SpO}_{2}$ overestimated $\mathrm{SaO}_{2}$, and a minimum $\mathrm{SpO}_{2}$ value of $96 \%$ to ensure $\mathrm{SaO}_{2}>90 \%$ was incorporated into a nurse-driven protocol [16]. In a study by van de Louw et al., large differences between $\mathrm{SpO}_{2}$ and $\mathrm{SaO}_{2}$ were found with poor $\mathrm{SpO}_{2}$ reproducibly. Both studies suggested an $\mathrm{SpO}_{2}$ above $94 \%$ to ensure $\mathrm{SaO}_{2}>90 \%[16,17]$. The accuracy of pulse oximetry was studied by Wilson et al. in a cohort of septic patients admitted to the emergency department. Their main finding was that pulse oximetry overestimated ABG-determined $\mathrm{SaO}_{2}$ by $2.75 \%$ [4]. When $\mathrm{SaO}_{2}$ needed to be determined, ABG analysis was recommended. A key problem in interpreting the findings reported in the literature on the use of pulse oximetry is that different kinds of pulse oximeters are used with different patented techniques for calculating $\mathrm{SpO}_{2}$, making comparisons difficult [18]. Corrections for the PFI have not been made in any of these studies.

Several possible explanations can be put forward to explain the disparity between the $\Delta$ Sat and the PFI values. Vasodilation in skin and muscle is not equally distributed in acid-base disorders. Higher $\mathrm{pH}$ results in vasodilation in the muscle but vasoconstriction in skin arteries where the $\mathrm{SpO}_{2}$ measurements are taken [19]. This skin vasoconstriction may result in lower $\mathrm{SpO}_{2}$ values and therefore the $\Delta$ Sat $\left(\mathrm{SpO}_{2}-\mathrm{SaO}_{2}\right)$ may decrease, resulting in a negative correlation. Moreover, a lower $\mathrm{pH}$ results in a lower $\mathrm{SaO}_{2}$ due to the rightward shift of the oxygen dissociation curve (Bohr Effect), resulting in an increased $\Delta$ Sat. Furthermore, in general and in isolated arteries, a lower $\mathrm{pH}$ results in vasodilation and therefore higher PFI values [19]. This association might result in a negative correlation. However, in our patient group a positive correlation was found. This might be explained by the fact that acidotic patients are frequently hemodynamically less stable, have a lower cardiac output, and are at risk for higher inotropic use. These factors might independently contribute to a lower PFI. In conclusion, many interacting factors may result in vasodilation or vasoconstriction. Our results have small correlation coefficients and may result in divergent values of blood oxygen levels.

To our knowledge, this is the first clinical study that correlated $\mathrm{SpO}_{2}$ accuracy with the PFI in adults. However, there are several limitations to our study. In our ICU, the ABG measurements were performed in our local central laboratory rather than with point-of-care equipment. A delay in the laboratory measurements could result in a lower arterial oxygen content and thus increased frequency of a positive $\mathrm{SpO}_{2}-\mathrm{SaO}_{2}$ difference. In our analyses this was not found, so we believe that this may not have influenced our results significantly. A second point of concern might be that we only used Philips pulse oximetry equipment, making headto-head comparison with other manufacturers and extrapolation to different clinical settings difficult. However, a study by Louie et al. demonstrated that the Philips, Masimo, and Nellcor pulse oximeters were similarly effective in detecting hypoxemia [18]. Furthermore, besides the surmised but not proven value concerning the reliability of the linear relationship between $\mathrm{SpO}_{2}$ and $\mathrm{SaO}_{2}$, PFI values have also been used to predict vasopressor requirements or mortality [2, 20-23]. Clearly, differences in patient population may account for this, and our study was neither powered nor designed to assess inotropic drug use or mortality. Another possible drawback of our study is that it was conducted in a single centre; however, bias can also be introduced easily in a multi-centre trial as equipment and laboratory procedures may differ slightly. 
Given that $\mathrm{SpO}_{2}$ does not reliably predict $\mathrm{SaO}_{2}$ values, despite in accordance to the manufacturer's precision (both around 2-3\%), the question arises as to how supplemental oxygen therapy can be adjusted without creating a hypoxic or hyperoxic state [11]. For example, in our ICU, we would rather be informed if the patient has an $\mathrm{SaO}_{2}$ of $89 \%$ instead of $93 \%$, whereas the corresponding monitor indicates $91 \%$. Moreover, our results imply that the PFI is not an accurate marker for $\mathrm{SaO}_{2}$ extrapolation and therefore will not be the primary determinant for adjusting the fraction of inspired oxygen $\left(\mathrm{FiO}_{2}\right)$ values to improve supplemental oxygen delivery. Hence, we suggest performing an ABG analysis to measure $\mathrm{SaO}_{2}$.

\section{Conclusions}

Our results indicate a clinically relevant discrepancy between $\mathrm{SaO}_{2}$ and $\mathrm{SpO}_{2}$ and only a small decrease in their difference in the presence of high arterial perfusion. However, even at high PFI values, differences in saturation can still be clinically significant. These findings may influence daily practice on how to adjust oxygen supply therapy based on $\mathrm{SpO}_{2}$ measurements only. In critical situations, we advise collecting ABG measurements instead of adjusting oxygen supply based on $\mathrm{SpO}_{2}$ values, regardless of the PFI values.

Author contributions MT selected the study design, acquired the data, drafted, and completed the manuscript for publication. LJ performed the statistical analysis, revised the manuscript, and approved it for publication. JN revised the manuscript and approved it for publication. NF selected the study design, revised the manuscript, gave the final approval for publication. All authors have read and approved the final manuscript.

\section{Compliance with ethical standards}

Conflict of interest The authors declare that they have no conflict of interest.

Open Access This article is distributed under the terms of the Creative Commons Attribution 4.0 International License (http://creativeco mmons.org/licenses/by/4.0/), which permits unrestricted use, distribution, and reproduction in any medium, provided you give appropriate credit to the original author(s) and the source, provide a link to the Creative Commons license, and indicate if changes were made.

\section{References}

1. Rhodes A, Evans LE, Alhazzani W, Levy MM, Antonelli M, Ferrer R, Kumar A, Sevransky JE, Sprung CL, Nunnally ME, Rochwerg B, Rubenfeld GD, Angus DC, Annane D, Beale RJ, Bellinghan GJ, Bernard GR, Chiche JD, Coopersmith C, De Backer DP, French CJ, Fujishima S, Gerlach H, Hidalgo JL,
Hollenberg SM, Jones AE, Karnad DR, Kleinpell RM, Koh Y, Lisboa TC, Machado FR, Marini JJ, Marshall JC, Mazuski JE, McIntyre LA, McLean AS, Mehta S, Moreno RP, Myburgh J, Navalesi P, Nishida O, Osborn TM, Perner A, Plunkett CM, Ranieri M, Schorr CA, Seckel MA, Seymour CW, Shieh L, Shukri KA, Simpson SQ, Singer M, Thompson BT, Townsend SR, Van der Poll T, Vincent JL, Wiersinga WJ, Zimmerman JL, Dellinger RP. Surviving sepsis campaign: international guidelines for management of sepsis and septic shock: 2016. Intensive Care Med. 2017;43:304-77. https://doi.org/10.1007/s00134-017-4683-6.

2. Hasanin A, Mukhtar A, Nassar H. Perfusion indices revisited. J Intensive Care. 2017;5:24. https://doi.org/10.1186/s4056 0-017-0220-5.

3. Jensen LA, Onyskiw JE, Prasad NG. Meta-analysis of arterial oxygen saturation monitoring by pulse oximetry in adults. Heart Lung. 1998;27:387-408.

4. Wilson BJ, Cowan HJ, Lord JA, Zuege DJ, Zygun DA. The accuracy of pulse oximetry in emergency department patients with severe sepsis and septic shock: a retrospective cohort study. BMC Emerg Med. 2010;10:9. https://doi.org/10.1186/1471-227X-10-9.

5. van Genderen ME, Bartels SA, Lima A, Bezemer R, Ince C, Bakker J, van Bommel J. Peripheral perfusion index as an early predictor for central hypovolemia in awake healthy volunteers. Anesth Analg. 2013;116:351-6. https://doi.org/10.1213/ ANE.0b013e318274e151.

6. Klijn E, Groeneveld AB, van Genderen ME, Betjes M, Bakker J, van Bommel J. Peripheral perfusion index predicts hypotension during fluid withdrawal by continuous Veno-venous hemofiltration in critically ill patients. Blood Purif. 2015;40:92-8. https:// doi.org/10.1159/000381939.

7. Lima AP, Beelen P, Bakker J. Use of a peripheral perfusion index derived from the pulse oximetry signal as a noninvasive indicator of perfusion. Crit Care Med. 2002;30:1210-3.

8. De Felice C, Latini G, Vacca P, Kopotic RJ. The pulse oximeter perfusion index as a predictor for high illness severity in neonates. Eur J Pediatr. 2002;161:561-2. https://doi.org/10.1007/s0043 1-002-1042-5.

9. Nitzan M, Romem A, Koppel R. Pulse oximetry: fundamentals and technology update. Med Devices (Auckl). 2014;7:231-9. https ://doi.org/10.2147/MDER.S47319.

10. Philips (2006) Intellivue Patiëntmonitor Mp70. M8000-9004g. Germany.

11. Philips. Reusable sensors that last durable and cost effective reusable $\mathrm{SpO}_{2}$ sensors. Eindhoven: Royal Philips Electronics; 2008.

12. Bland JM, Altman DG. Statistical methods for assessing agreement between two methods of clinical measurement. Lancet. 1986;1:307-10.

13. Hо KM. Using linear regression to assess dose-dependent bias on a bland-altman plot. J Emerg Crit Care Med. 2018;2:68.

14. General Assembly of the World Medical A. World Medical Association Declaration of Helsinki: ethical principles for medical research involving human subjects. J Am Coll Dent. 2014;81:14-8.

15. Perkins GD, McAuley DF, Giles S, Routledge H, Gao F. Do changes in pulse oximeter oxygen saturation predict equivalent changes in arterial oxygen saturation? Crit Care. 2003;7:R67. https://doi.org/10.1186/cc2339.

16. Seguin P, Le Rouzo A, Tanguy M, Guillou YM, Feuillu A, Malledant Y. Evidence for the need of bedside accuracy of pulse oximetry in an intensive care Unit. Crit Care Med. 2000;28:703-6.

17. Van de Louw A, Cracco C, Cerf C, Harf A, Duvaldestin P, Lemaire F, Brochard L. Accuracy of pulse oximetry in the intensive care unit. Intensive Care Med. 2001;27:1606-13. https://doi. org/10.1007/s001340101064.

18. Louie A, Feiner JR, Bickler PE, Rhodes L, Bernstein M, Lucero J. Four types of pulse oximeters accurately detect hypoxia during 
low perfusion and motion. Anesthesiology. 2018;128:520-30. https://doi.org/10.1097/ALN.0000000000002002.

19. Mitchell JH, Wildenthal K, Johnson RL Jr. The effects of acidbase disturbances on cardiovascular and pulmonary function. Kidney Int. 1972;1:375-89.

20. Rasmy I, Mohamed H, Nabil N, Abdalah S, Hasanin A, Eladawy A, Ahmed M, Mukhtar A. Evaluation of perfusion index as a predictor of vasopressor requirement in patients with severe sepsis. Shock. 2015;44:554-9. https://doi.org/10.1097/SHK.0000000000 000481.

21. Janak JC, Howard JT, Goei KA, Weber R, Muniz GW, HinojosaLaborde C, Convertino VA. Predictors of the onset of hemodynamic decompensation during progressive central hypovolemia: comparison of the peripheral perfusion index, pulse pressure variability, and compensatory reserve index. Shock. 2015;44:548-53. https://doi.org/10.1097/SHK.0000000000000480.
22. He H, Long Y, Liu D, Wang X, Zhou X. Clinical classification of tissue perfusion based on the central venous oxygen saturation and the peripheral perfusion index. Crit Care. 2015;19:330. https ://doi.org/10.1186/s13054-015-1057-8.

23. He HW, Liu DW, Long Y, Wang XT. The peripheral perfusion index and transcutaneous oxygen challenge test are predictive of mortality in septic patients after resuscitation. Crit Care. 2013;17:R116. https://doi.org/10.1186/cc12788.

Publisher's Note Springer Nature remains neutral with regard to jurisdictional claims in published maps and institutional affiliations. 